\title{
Evolution of Intermediate-Mass Black Hole X-Ray Binaries
}

\author{
Xiang-Dong Li \\ Department of Astronomy, Nanjing University, Nanjing 210093, P. R. China \\ lixd@nju.edu.cn
}

\begin{abstract}
The majority of the ultraluminous X-ray sources (ULXs) in external galaxies are believed to be accreting black holes in binary systems; some of the black holes could be as massive as $\sim 100-1000 M_{\odot}$. We have performed evolution calculations for intermediate-mass black hole X-ray binaries, assuming they are formed in dense star clusters via tidal capture. The results are compared with those for stellar-mass black holes X-ray binaries. We find that these two types of black holes may have similar companion stars and binary orbits if observed as ULXs. However, intermediate-mass black holes seem to be favored in explaining the most luminous ULXs. We also discuss the possibilities of transient behavior and beamed emission in the evolution of these binary systems.
\end{abstract}

Subject headings: accretion, accretion disks - binaries: close - X-rays: binaries

\section{Introduction}

Ultraluminous X-ray sources (ULXs) are point-like extra-nuclear X-ray sources found in nearby galaxies, with (isotropic) X-ray luminosities in excess of $10^{39} \mathrm{ergs}^{-1}$, roughly corresponding to the Eddington limit accretion luminosity for a $10 M_{\odot}$ star (Fabbiano 1989). The majority of ULXs are believed to be black holes accreting from their binary companion stars (see, e.g., reviews by Fabbiano \& White 2004; Colbert \& Miller 2004), or in few cases, perhaps from the fallback material originating from supernovae that have produced these sources (Li 2003). Many works have shown that stellar-mass black holes (SMBHs) can account for the properties of most ULXs, but for the most luminous ones (with X-ray luminosities $L_{\mathrm{X}} \gtrsim 10^{40} \mathrm{erg} \mathrm{s}^{-1}$ ), assumptions of either truly super-Eddington X-ray emission (Begelman 2002), or anisotropic (beamed) emission (King et al. 2001; Körding, Falcke, \& Markoff 2002) are required. Alternatively, the latter sources could be intermediate-mass black holes (IMBHs; Colbert \& Mushotzky 1999). This suggestion seems to be supported by 
their relatively low disk color temperatures inferred from X-ray spectral analysis (e.g. Miller, Fabian, \& Miller 2004).

It is interesting and important to discriminate between these two kinds of black holes observationally. Kalogera et al. (2004) suggested that IMBHs in X-ray binaries are more likely to be transient due to the thermal-viscous instability (King, Kolb, \& Burderi 1996). The reason is that, both the high masses and wide orbits of IMBHs lead to a larger accretion disk and hence lower temperature in the disk compared to the SMBH case. In this Letter, with different assumptions on IMBH binary formation from Kalogera et al. (2004), we have calculated the evolution of IMBH X-ray binaries with a massive donor star. We describe the initial parameters for the binary evolution and the calculated results in section 2 and 3 respectively. In section 4 we discuss the relation between IMBHs, SMBHs, and ULXs. Our conclusions are summarized in section 5 .

\section{Assumptions on the formation of IMBH X-ray binaries}

We consider IMBHs as ULXs associated with star formation regions in spiral or irregular galaxies. In this situation, an IMBH could be formed through runaway collision in dense young star clusters within $\sim 3$ Myr (Portegies Zwart et al. 2004). It may acquire a binary companion via exchange encounters and/or tidal capture in the host cluster. The latter process was recently investigated by Hopman, Portegies Zwart, \& Alexander (2004). Some of their results were adopted in our evolution calculations.

The BH masses were taken to be $\sim 100-1000 M_{\odot}-$ more massive BHs were not considered here. The reasons are as follows. First, both spectral analysis (e.g. Miller et al. 2003; Wang et al. 2004; Di Stefano et al. 2004) and numerical calculations (Portegies Zwart et al. 2004) have revealed IMBH masses within this range. Second, successful circularization of binaries formed by tidal capture is only possible provided that the $\mathrm{BH}$ masses are less than a few thousand $M_{\odot}$ (Hopman, Portegies Zwart, \& Alexander 2004).

The masses of the donor stars were assumed to be $\sim 5-20 M_{\odot}$. These massive stars sink to the center of the clusters together with the $\mathrm{BH}$, and are more likely to be captured by a $\mathrm{BH}$ because of their large cross section and close spatial distribution around the $\mathrm{BH}$ (Kalogera et al. 2004).

After in-spiral and circulation, the binary separation can be derived from orbital angular momentum conservation to be (Hopman, Portegies Zwart, \& Alexander 2004),

$$
a \sim(4-5)\left(M_{\mathrm{BH}} / M\right)^{1 / 3} R
$$


where $M_{\mathrm{BH}}$ is the $\mathrm{BH}$ mass, $M$ and $R$ the mass and radius of the companion star, respectively. The above equation implies that the stellar radius is roughly half of its Roche-lobe radius, i.e., the orbital periods of the incipient binaries are around $2-4$ days.

\section{Results of binary evolution calculations}

We have followed the evolution of the binary systems containing an IMBH and a massive donor star for the initial parameters given in last section, using an updated version of the evolution code developed by Eggleton (1971). The opacities in the code are from Rogers \& Iglesias (1992), and from Alexander \& Ferguson (1994) for temperatures below $10^{3.8} \mathrm{~K}$. For the donor star we assumed a solar chemical composition $(X=0.7, Y=0.28, Z=0.02)$ and a mixing length parameter $\alpha=2$. To follow the details of mass transfer process, we included losses of orbital angular momentum due to mass loss and gravitational wave radiation. We limited the mass accretion rate of the black hole to its Eddington limit rate, and let the excess mass be lost from the system with the specific orbital angular momentum of the black hole. We also assumed that the companion stars are on zero-age main-sequence when they have been captured and settled in a circular orbit. This means that the time for circular IMBH binary formation is much less than the stellar main-sequence lifetime. This may not be true for the companion stars more massive than $\sim 15 M_{\odot}$, since the formation history of IMBH binaries could be as long as $\sim 10^{7}$ yrs (Portegies Zwart et al. 2004). So our results for stars of $M \gtrsim 15 M_{\odot}$ should be regarded as the most optimistic cases.

Figure 1 shows two examples of mass transfer sequences for a binary containing a $1000 M_{\odot} \mathrm{BH}$ with a 5 and $15 M_{\odot}$ donor star, respectively [changing the $\mathrm{BH}$ masses (say, to $100 M_{\odot}$ ) does not alter the results considerably]. In the figure the mass transfer rates have been converted into X-ray luminosities to be compared with observations. The X-ray luminosities were calculated according to the slim disk model by Ohsuga et al. (2002), in which photon trapping effect was included ${ }^{1}$. The solid and dashed curves correspond respectively to stable and unstable mass transfer in the accretion disk, according to the criterion given in Dubus et al. (1999).

Since the initial binary orbit is too wide for the companion star to fill its Roche lobe, the mass transfer through Roche-lobe overflow begins until the star evolves and expands after a time labelled below the time-axis in the figure. The X-ray luminosities are generally around $10^{40} \mathrm{erg} \mathrm{s}^{-1}$, comparable with those of the most luminous ULXs. However, the stable

\footnotetext{
${ }^{1}$ We fit the numerical results in (more realistic) model B in Ohsuga et al. (2002) by the formula $L_{\mathrm{X}} / L_{\mathrm{E}} \simeq$ $0.1 \dot{m} /\left(1+\dot{m}^{1 / 2} / 3\right)$, where $L_{\mathrm{E}}$ is the Eddington luminosity, and $\dot{m}=\dot{M} c^{2} / L_{\mathrm{E}}$.
} 
$\mathrm{X}$-ray lifetime is generally $\sim 10^{6} \mathrm{yrs}$, much shorter than the main-sequence lifetime of the donor stars. Transient behavior usually occurs when the orbits become sufficiently wide. The absence of extreme long-term variability in ULXs suggests that they are likely to be persistent X-ray sources (Roberts et al. 2004). If it is correct, IMBHs can be observed as ULXs at the former part of their evolutions [at later time the mass transfer rates can also be very high when the donor star is on (super)giant branch, but with very short duration, see Rappaport, Podsiadlowski, \& Pfahl (2004)]. There is a dip/gap in the mass transfer rates when the core hydrogen in the star exhausts and the star deviates from thermal equilibrium. Similar features have already been found in the calculations by Kalogera et al. (2004), Podsiadlowski, Rappaport, \& Han (2003), and Rappaport, Podsiadlowski, \& Pfahl (2004).

For comparison, we show in Fig. 2 the mass transfer sequences for typical SMBH X-ray binaries. The BH mass was assumed to be $10 M_{\odot}$. The mass of the donor star was also taken to be 5 and $15 M_{\odot}$, respectively. In each case we considered case A and B mass transfer. It can be seen that there is sufficiently long time $\left(\sim 10^{7}-10^{8}\right.$ yrs $)$ for these binaries to appear as persistent ULXs with $L_{\mathrm{X}} \sim 10^{39} \mathrm{erg} \mathrm{s}^{-1}$, but the X-ray lifetime with $L_{\mathrm{X}} \gtrsim 10^{40} \mathrm{erg} \mathrm{s}^{-1}$ is around a few $10^{4}-10^{6} \mathrm{yrs}$, comparable with and even shorter than that for IMBHs.

Figure 3 summarizes the evolutions of massive IMBH X-ray binaries. Various symbols have been used to describe the states of the binaries: stars, rectangles and triangles represent $L_{\mathrm{X}} \gtrsim 10^{40} \mathrm{erg} \mathrm{s}^{-1}, 10^{39} \mathrm{erg} \mathrm{s}^{-1}<L_{\mathrm{X}}<10^{40} \mathrm{erg} \mathrm{s}^{-1}$, and $L_{\mathrm{X}} \lesssim 10^{39} \mathrm{erg} \mathrm{s}^{-1}$, filled and open symbols indicate stable and unstable mass transfer state, respectively. The figure demonstrates that IMBH binaries, if observed as persistent ULXs, have relatively massive companions in narrow orbits. This feature is quite similar to those for SMBH X-ray binaries (Podsiadlowski, Rappaport, \& Han 2003; Rappaport, Podsiadlowski, \& Pfahl 2004)

\section{Discussion}

The purpose of this work is to examine whether the hypothesized IMBHs can reproduce the observed properties of (some of) the ULXs. There have been several works on this subject in the literature. In the binary evolution calculations we have used the results in Hopman, Portegies Zwart, \& Alexander (2004) on tidal capture to determine the initial conditions for the incipient IMBH binaries. But we allowed stellar expansion before Roche-lobe overflow, and got the following results different from theirs: (1) the donor stars have already evolved off main-sequence when mass transfer begins; (2) the X-ray lifetime for IMBHs as ULXs is around $10^{6} \mathrm{yr}$, much shorter than the main-sequence lifetime of the donor stars. These can present constraints on the donor stars of IMBH-ULXs. For example, the age of the star cluster MGG-11 in the irregular galaxy M82 is $7-12$ Myr. If the ULX M82 X-7 in 
this cluster (Matsumoto \& Tsuru 1999) is powered by an IMBH of mass $\sim 1000 M_{\odot}$, a $10-15 M_{\odot}$ donor star is required to account for the luminosity and age of the ULX. In a more recent paper, Portegies Zwart, Dewi, \& Maccarone (2004) performed detailed binary evolution calculations of $2-15 M_{\odot}$ stars which transfer mass to a $100-2000 M_{\odot} \mathrm{BH}$. These authors initialized the binary systems with an (arbitrarily) selected orbital period at birth (ranging from less than a day to a few hundred days). Their calculated mass transfer rates and time with initial orbital period of the order of a day are in general agreement with ours.

Systematic studies on evolution of SMBH X-ray binaries have been carried by Podsiadlowski, Rappaport, \& Han (2003) and Rappaport, Podsiadlowski, \& Pfahl (2004). The results in Fig. 2 can be regarded as individual cases of their population synthesized results. We notice that different parameterization for estimating the X-ray luminosities has been adopted here and in their works. Rappaport, Podsiadlowski, \& Pfahl (2004) assumed an energy conversion coefficient depending on the BH spin and let $L_{\mathrm{X}}$ be as high as 10 times of the Eddington luminosity, as suggested by Begelman (2002). We have used the results of Ohsuga et al. (2002), who calculated the emission from the supercritical accretion disk, considering the photon trapping effect. This difference causes the mass transfer rate to be about 10 times higher in our work (i.e., $\dot{M} \sim 10^{-5} M_{\odot} \mathrm{yr}^{-1}$ ) than in Rappaport, Podsiadlowski, \& Pfahl (2004), for $L_{\mathrm{X}}$ to reach $10^{40} \mathrm{erg} \mathrm{s}^{-1}$, for a $10 M_{\odot} \mathrm{BH}$. The duration of the high-luminosity X-ray emission phase is correspondingly $\sim 10$ times shorter to be $\sim 10^{6}$ yrs for a massive donor star.

From evolution calculations we have shown that, as ULXs, both IMBH and SMBH Xray binaries actually have similar characteristics in their companions stars and binary orbits, which make it difficult to discriminate them observationally. However, if the donor star is more massive than $\sim 10 M_{\odot}$, the IMBH binaries are most likely to reside deeply inside the host star cluster, while SMBH binaries generally have been "kicked" outside (Zezas et al. 2002). For an intermediate- or low-mass donor star, the IMBH binaries could be hostless, since the preceding time before mass transfer could be long enough for the cluster to be tidally dispersed, if it is close to the galactic center (Portegies Zwart 2004).

For the most luminous ULXs with $L_{\mathrm{X}} \gtrsim 10^{40} \mathrm{erg} \mathrm{s}^{-1}$, a potential problem related to the SMBH model is that, according to Ohsuga et al. (2002), the effective temperatures of supercritical accretion disks with $\dot{M} \sim 10^{-5} M_{\odot} \mathrm{yr}^{-1}$ are still as high as $\sim 1 \mathrm{keV}$, inconsistent with spectral analyzed results for these sources (e.g. Miller, Fabian, \& Miller 2004). It is not known how the temperature profile is in the Begelman (2002) disk model. On the other hand, IMBHs have the advantage of low disk temperatures because of their high masses.

We also find that transient behavior is a common feature in late evolutionary stages in these two types of binaries. It may be difficult to tell IMBHs from SMBHs by transient 
behavior, as suggested by Kalogera et al. (2004). The differences between our work and Kalogera et al. (2004) result from the following facts. (1) We have adopted the BH masses no more than $1000 M_{\odot}$ in the calculations. With these values, one can actually draw from Fig. 1 in Kalogera et al. (2004) the similar results as ours. (2) We assumed tidal capture as the main formation channel of IMBH binaries, from which we got the initial orbital periods of $\sim 2-4$ days; Kalogera et al. (2004) favor orbital periods in excess of $\sim 100$ days, but the duration of the mass transfer episode would decrease to be very short (less than a few $10^{4}$ yrs), making them difficult to be observed.

It should be noted that the calculated mass transfer rates are long-term, averaged ones. It is unclear how to relate these secular mass transfer rates to observable instantaneous X-ray luminosities, and to disk instability influenced by (short-term) mass transfer and Xray irradiation. Moreover, the mechanisms and criterion for the thermal-viscous instability in irradiated accretion disk are not yet fully understood. It is premature to predict the instability occurrence from only calculated mass transfer rates.

Another interesting feature is that, if anisotropic (or beamed) emission is associated with mass transfer rates comparable to the Eddington rate, as suggested before (e.g. King et al. 2001), our calculations indicate that IMBHs may also have anisotropic emission, since the mass transfer rates can be sufficiently high to satisfy the condition above. However, anisotropic X-ray emission is not preferred in our opinion for persistent ULXs, since the most important stabilizing factor for disk instability, the efficiency of X-ray irradiation, will be greatly reduced if the emission is beamed (usually in the direction perpendicular to the disk plane), and the ULXs would become transient sources.

\section{Summary}

We have calculated the evolutionary sequences of IMBH X-ray binaries formed through tidal capture in dense star clusters, and compared the results with those of SMBH binaries. We found that IMBHs seem to be capable of explaining the nature of most luminous ULXs, and their companion stars and binary orbits could be similar to those of SMBH-ULXs. We suggest that transient behavior and beamed emission may be not enough to distinguish between IMBHs and SMBHs.

I would like to thank Ranold Webbink for helpful discussion, and the referee, Philipp Podsiadlowski for clarifying comments. This work was supported by NSFC through grant number 10025314 and MSTC through grant number NKBRSF G19990754. 


\section{REFERENCES}

Alexander, D. R. \& Ferguson, J. W. 1994, ApJ, 437, 879

Begelman, M. C. 2002, ApJ, 568, L97

Colbert, E. J. M. \& Miller, M. C. 2004, talk at the Tenth Marcel Grossmann Meeting on General Relativity, Rio de Janeiro, July 20-26, 2003. Proceedings edited by M. Novello, S. Perez-Bergliaffa and R. Ruffini, World Scientific, Singapore, 2004 [astro$\mathrm{ph} / 0402077]$.

Colbert, E. J. M. \& Mushotzky, R. F. 1999, ApJ, 519, 89

Di Stefano, R., Primini, F. A., Kong, A. K. H., \& Russo, T. 2004, preprint [astro-ph/0405238]

Dubus, G., Lasota, J.-P., Hameury, J.-M., \& Charles, P. 1999, MNRAS, 303, 139

Eggleton, P. P. 1971, MNRAS, 151, 351

Fabbiano, G. 1989, ARA\&A, 27, 87

Fabbiano, G. \& White, N. E. 2004, to appear in "Compact Stellar X-ray Sources", eds. W. H. G. Lewin and M. van der Klis (Cambridge Univ. Press, Cambridge) [astro$\mathrm{ph} / 0307077]$

Hopman, C. Portegies Zwart, S. F., \& Alexander, T. 2004, ApJ, 604, L101

Kalogera, V., Henninger, M., Ivanova, N. \& King, A. R. 2004, ApJ, 603, L41

King, A. R., Davies, M. B., Ward, M. J., Fabbiano, G., \& Elvis, M. 2001, ApJ, 552, L109

King, A. R., Kolb, U., \& Burderi, L. 1996 ApJ, 464, L127

Körding, E., Falcke, H., \& Markoff, S. 2002, A\&A, 382, L13

Li, X.-D. 2003, ApJ, 596, L199

Matsumoto, H. \& Isuru, T. G. 1999, PASJ, 51, 321

Miller, J. M., Fabbiano, G., Miller, M. C., \& Fabian, A. C. 2003, ApJ, 585, L37

Miller, J. M., Fabian, A. C., \& Miller, M. C. 2004, preprint [astro-ph/0406656]

Ohsuga, K., Mineshige, S., Mori, M., \& Umemura, M. 2002, ApJ, 574, 315

Podsiadlowski, P., Rappaport, S., \& Han, Z. 2003, MNRAS, 341, 385 
Portegies Zwart, S. 2004, to appear in "Joint Evolution of Black Holes and Galaxies" of the Series in High Energy Physics, Cosmology and Gravitation, eds M. Colpi, V.Gorini, F.Haardt and U.Moschella (IOP Publishing, Bristol and Philadelphia) [astro-ph/0406550]

Portegies Zwart, S. F., Baumgardt, H., Hut, P., Makino, J., \& McMillan, L. W. 2004, Nat, 428,724

Portegies Zwart, S. F., Dewi, J., \& Maccarone, T. 2004, preprint [astro-ph/0408402]

Rappaport, S., Podsiadlowski, P., \& Pfahl, E. 2004, preprint [astro-ph/0408032]

Roberts, T. P., Warwick, R. S., Ward, M. J., \& Goad, M. R. 2004, MNRAS, 349, 1193

Rogers, F. J. \& Iglesias, C. A. 1992, ApJS, 79, 597

Wang, Q. D., Yao, Y., Fukui, W., Zhang, S. N., \& Williams, R. 2004, ApJ, 609, 113

Zezas, A., Fabbiano, G., Rots, A. H., \& Murray, S. S. 2002, ApJ, 577, 710 

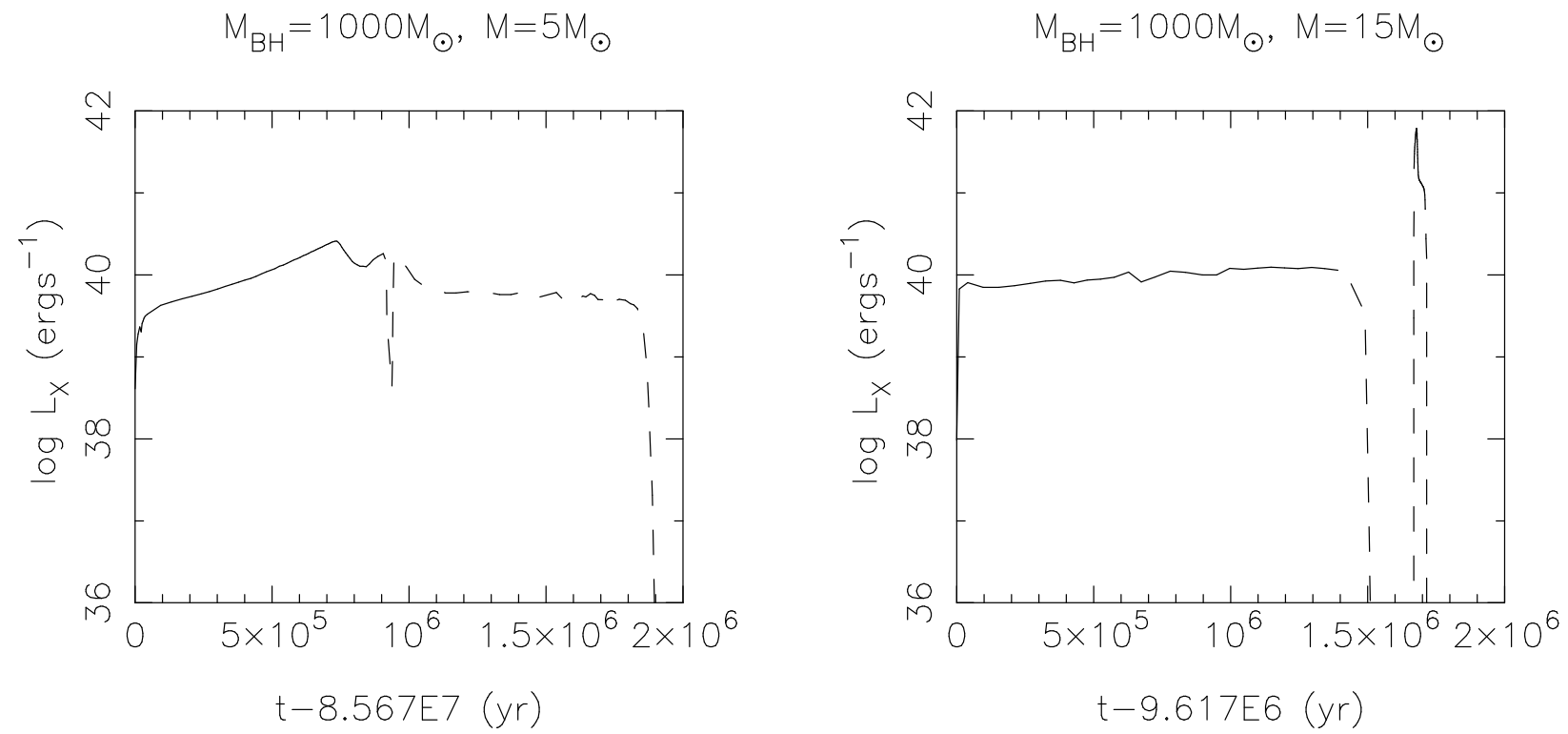

Fig. 1.- Potential X-ray luminosities as a function of time for two IMBH X-ray binary evolution sequences. The duration of the evolutionary phase before Roche-lobe overflow has been subtracted from the real time. The solid and dashed curves reflect thermal-viscously stable and unstable mass transfer, respectively. 
$M_{B H}=10 M_{\odot}, M=5 M_{\odot}$

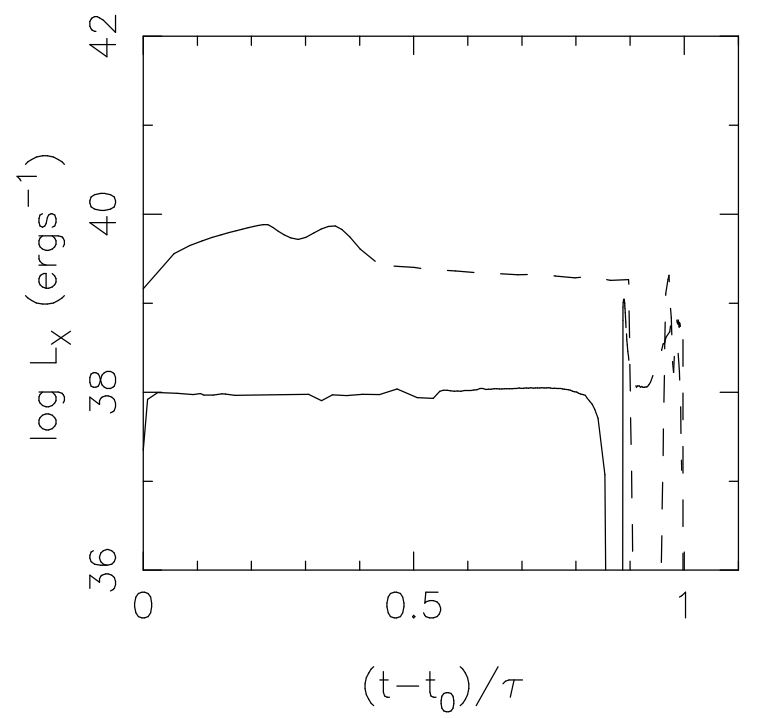

$M_{\mathrm{BH}}=10 \mathrm{M}_{\odot}, \quad M=15 \mathrm{M}_{\odot}$

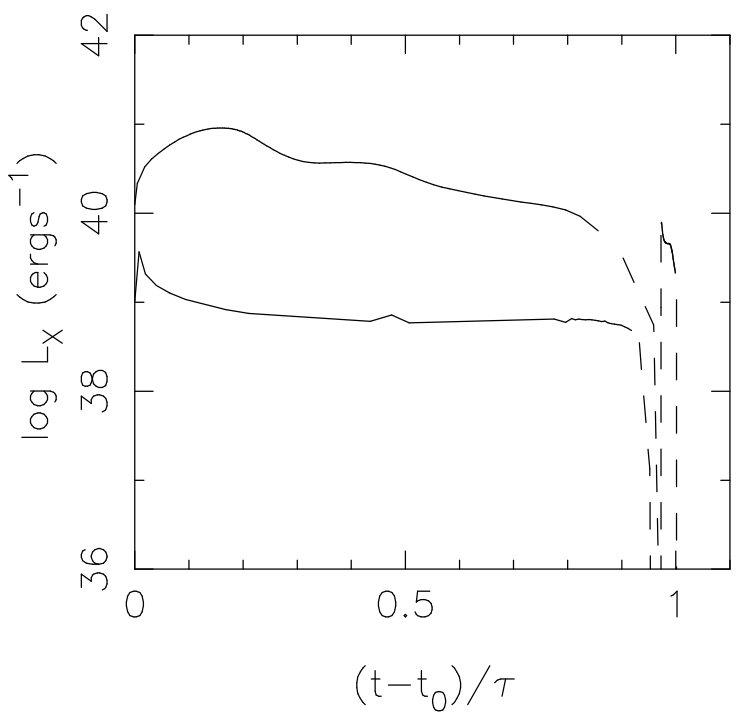

Fig. 2.- Potential X-ray luminosities as a function of normalized time for four SMBH Xray binary evolution sequences. The solid and dashed curves reflect thermal-viscously stable and unstable mass transfer, respectively. In the left panel the parameters for the upper and lower curve are: the initial binary orbital periods $P_{\text {orb,i }}=3.43,0.87 \mathrm{~d}$, the time at the onset of mass transfer $t_{0}=1.0 \times 10^{8}, 3.8 \times 10^{7} \mathrm{yr}$, and the duration of total mass transfer episode $\tau=1.2 \times 10^{6}, 1.4 \times 10^{8} \mathrm{yr}$, respectively. In the right panel: $P_{\text {orb,i }}=3.17,0.65 \mathrm{~d}$; $t_{0}=1.1 \times 10^{7}, 2.5 \times 10^{5} \mathrm{yr} ; \tau=4.4 \times 10^{4}, 3.1 \times 10^{7} \mathrm{yr}$. 


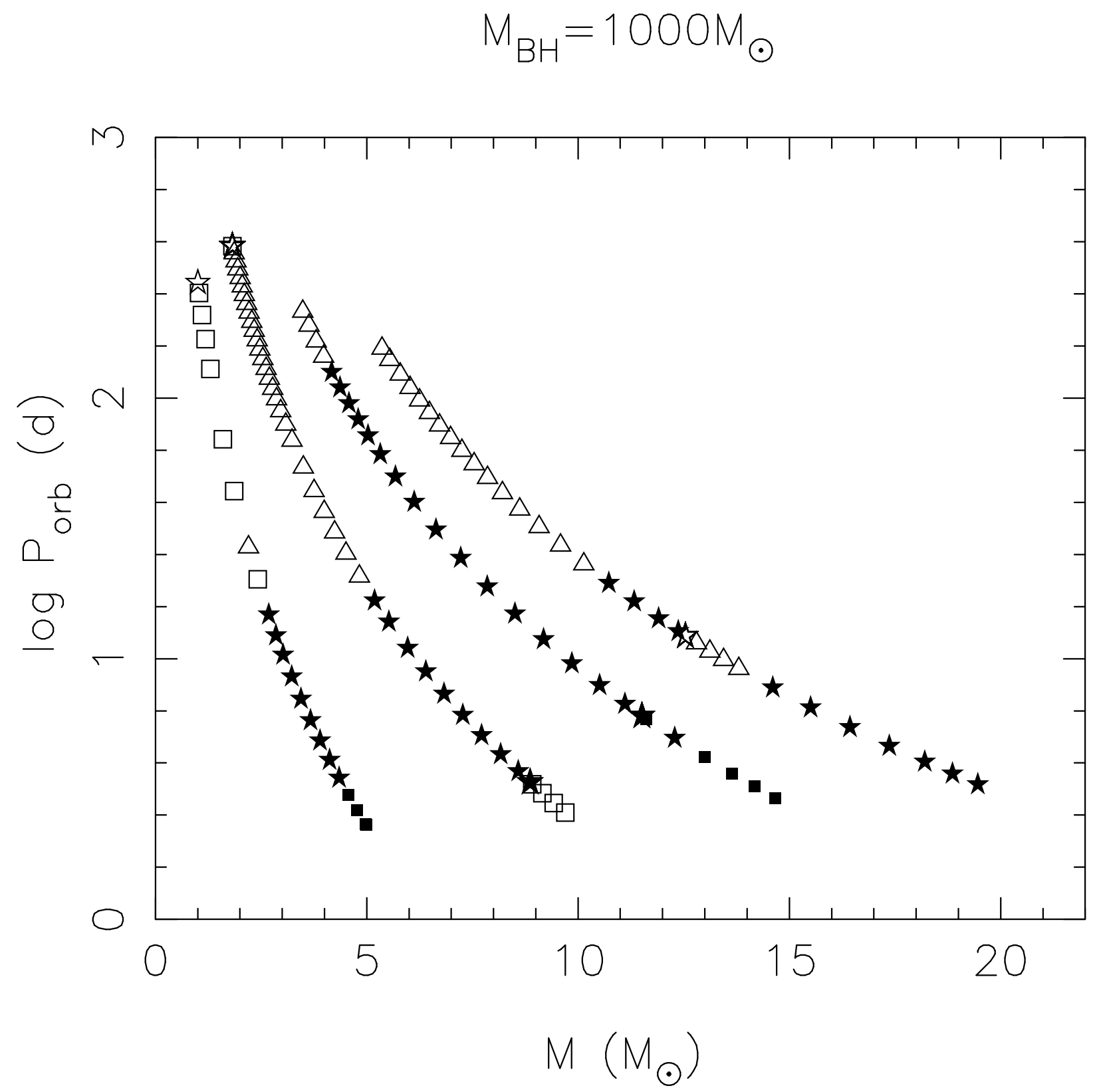

Fig. 3.- IMBH binary evolution sequences on the donor mass - orbital period plane. See text for the meanings of various symbols 